\title{
Thermodynamics of Polymer-Polymer-Solvent and Block Copolymer-Solvent Systems \\ II. Theoretical Treatment of Data with the Nonrandom New Flory Theory
}

\author{
C. Panayiotou* and J. H. Vera \\ Department of Chemical Engineering, McGill University, \\ Montreal, Quebec, Canada, H3A $2 A 7$ \\ (Received August 18, 1983)
}

\begin{abstract}
Experimental data previously reported ${ }^{1}$ are treated with the nonrandom new Flory theory of polymer solutions. The theory is applied to polymer-polymer-solvent systems and extended to the block copolymer-solvent case. Polymer-polymer binary interaction parameters are determined. Equations for $\chi$ interaction parameters are identical in form for the polymer-solvent, polymer-polymer-solvent and block copolymer-solvent case. The ability of the theory to fit the experimental data is satisfactory.
\end{abstract}

KEY WORDS Polymer Mixtures / Block Copolymers / New Flory Theory / Nonrandom Mixing /

Most of the reported polymer pairs ${ }^{2,3}$ showing an appreciable degree of compatibility are by no means simple nonpolar molecules. Specific interactions imply a certain degree of nonrandomness in molecular arrangements in the mixture, although they are not, probably, the only cause for nonrandom arrangements in liquids and their mixtures. Orientational order has been observed even in $n$-alkane liquids. ${ }^{4}$ The need for corrections for nonrandomness was suspected several times in the past ${ }^{5-7}$ partly as a cause of discrepancies between theoretical and experimental results.

In this work, we treat the experimental data reported previously ${ }^{1}$ with the equation of state theory of Flory and coworkers ${ }^{8}$ corrected for nonrandomness. The correction for nonrandomness follows closely the lines of our previous work. ${ }^{7,9}$ Working equations are presented for ternary systems and the theory is extended to include the solvent-block copolymer case. The new Flory theory ${ }^{8}$ has been chosen because of its simplicity and the fact that the pure component equation of state parameters are frequently available in the literature.

\section{POLYMER-POLYMER-SOLVENT CASE}

The configurational partition function (the other factors of the partition function are of no concern to us here) in its maximum term approximation may be written as ${ }^{8,9}$

$$
Q_{\mathrm{N}}=g_{\mathrm{c}} g_{\mathrm{NR}} \psi^{N} \exp \left(-E_{0} / R T\right)
$$

where $g_{\mathrm{c}}$ is the combinatorial contribution accounting for the number of distinguishable random arrangements of the molecules in the system; $g_{\mathrm{NR}}$ is a correction to $g_{\mathrm{c}}$ for nonrandom arrangements. $\psi$ is the "cell" partition function or otherwise the "free volume" contribution of each of the $N$ molecules; $E_{0}$ is the mean intermolecular (attractive) energy of the system; $R$, gas constant and $T$, the

\footnotetext{
* Present address: Department of Chemical Engineering, University of Salonica, Greece.
} 
temperature.

For simplicity, we keep for $g_{\mathrm{c}}$ the approximation of Flory ${ }^{10}$ for a system of $N_{1}$ monomers, $\cdots, N_{i} r_{i}$-mers, namely

$$
g_{\mathrm{c}}=\frac{(r N) !}{\prod_{i} N_{i} !}\left(\frac{Z-1}{r N}\right)^{N(r-1)}
$$

where

$$
r N=\sum_{i} N_{i} r_{i}
$$

and

$$
N=\sum_{i} N_{i}
$$

The quasi-lattice coordination number $Z$ has been arbitrarily set equal to ten.

For the free volume term, we assume

$$
\psi=\left[\gamma v^{*}\left(\tilde{v}^{1 / 3}-1\right)^{3}\right]^{r c}
$$

with

$$
r c=\sum \phi_{i} r_{i} c_{i}
$$

$3 r_{i} c_{i}$ are the equivalent number of external degrees of freedom per $r_{i}$-mer in the Prigogine $^{11}$ sense.

If $N_{i j}$ is the total number of $i-j$ contact pairs in the system then, in the van der Waalstype energy interaction form the intermolecular energy of the system may be written as

$$
-E_{0}=\sum_{j \geqslant i} \frac{N_{i j} \varepsilon_{i j}}{v}
$$

where $\varepsilon_{i j}$ is an interactional constant characteristic of pair $i-j$.

The quasi-chemical ${ }^{12}$ correction for nonrandomness may be written as:

$$
g_{\mathrm{NR}}=\frac{\prod_{i} N_{i i}^{0} ! \prod_{j \geqslant i}\left[\frac{N_{i j}^{0}}{2} !\right]^{2}}{\sum_{i} N_{i i} ! \prod_{j \geqslant i}\left[\frac{N_{i j}}{2} !\right]^{2}}
$$

where superscript 0 indicates random values of $N_{i j}$ 's.

The segment fraction is defined as

$$
\phi_{i}=\frac{N_{i} r_{i}}{\sum_{j} N_{j} r_{j}}
$$

For an $r_{i}$-mer it is assumed that the number of intermolecular contacts in which molecule $i$ participates is given by ${ }^{12}$

$$
Z q_{i}=(Z-2) r_{i}+2
$$

The surface fraction $\theta_{i}$ is given by

$$
\theta_{i}=\frac{N_{i} Z q_{i}}{N Z q}
$$

where

$$
N Z q=\sum_{i} N_{i} Z q_{i}
$$

Due to nonrandom arrangements, the local surface fractions $\theta_{i j}$ are different from the overall surface fractions given by eq 11 . Thus, the total number of $i-i$ contacts is approximated as

$$
N_{i i}=\frac{N_{i} Z q_{i}}{2} \theta_{i i}
$$

and, similarly, for $i-j$ contacts

$$
N_{i j}=N_{i} Z q_{i} \theta_{j i}=N_{j} Z q_{j} \theta_{i j}
$$

$\theta_{i i}$ is the fraction of nearest neighbors of type $i$ around a central segment of type $i$ and $\theta_{i j}$ is the fraction of nearest neighbors of type $i$ around a central segment of type $j$. The factor $1 / 2$ in eq 13 is necessary in order to account for the symmetry of the arrangement $i-i$. In terms of nonrandom factors, the above local surface fractions may be written as

$$
\begin{aligned}
& \theta_{i i}=\theta_{i} \Gamma_{i i} \\
& \theta_{i j}=\theta_{i} \Gamma_{i j} \\
& \theta_{j i}=\theta_{j} \Gamma_{i j}
\end{aligned}
$$

In contrast to $\theta_{i j}$ 's the nonrandom factors $\Gamma_{i j}$ 's have no directional character $\left(\Gamma_{i j}=\Gamma_{j i}\right)$.

Following Guggenheim's quasi-chemical treatment, ${ }^{12}$ the values of the $N_{i j}$ 's $(i \neq j)$ are those that minimize the free energy of the system, leading to the quasi-chemical 
conditions $^{9}$

$$
\frac{\theta_{i i} \theta_{j j}}{\theta_{i j} \theta_{j i}}=\exp \frac{\varepsilon_{i i}+\varepsilon_{j j}-2 \varepsilon_{i j}}{v R T}=G_{i j}
$$

In the case of ternary systems, there are three quasi-chemical conditions, eq 16. In terms of local surface area fractions, the energy term $-E_{0}$ may be written for the ternary case as:

$$
\begin{aligned}
-E_{0}= & \frac{N r v^{*}}{v}\left(\phi_{1} P_{1}^{*}+\phi_{2} P_{2}^{*}+\phi_{3} P_{3}^{*}\right. \\
& \left.-\phi_{1} \theta_{21} X_{12}-\phi_{2} \theta_{32} X_{23}-\phi_{3} \theta_{13} X_{31}\right)
\end{aligned}
$$

where

$$
P_{i}^{*}=\frac{Z q_{i}}{2 r_{i}} \frac{\varepsilon_{i i}}{v^{* 2}}
$$

and

$$
X_{i j}=\frac{Z q_{i}}{2 r_{i}} \frac{\varepsilon_{i i}+\varepsilon_{j j}-2 \varepsilon_{i j}}{v^{* 2}} \quad(i \neq j)
$$

The characteristic pressure, $P^{*}$, of the system is then given by

$$
\begin{aligned}
P^{*}= & \phi_{1} P_{1}^{*}+\phi_{2} P_{2}^{*}+\phi_{3} P_{3}^{*}-\phi_{1} \theta_{21} X_{12} \\
& -\phi_{2} \theta_{32} X_{23}-\phi_{3} \theta_{13} X_{31}
\end{aligned}
$$

while the characteristic temperature $T^{*}$ is obtained from the relation

$$
P^{*} v^{*}=c R T^{*}
$$

Each of the three nonrandom factors $\Gamma_{i j}(i \neq j)$ is given by a relation of the form

$$
\begin{aligned}
& \Gamma_{i j}= \\
& \frac{2\left(1-2 \lambda \theta_{t}\right)}{\left(1-2 \theta_{t}\right)+\left[\left(1-2 \theta_{t}\right)^{2}-4\left(1-G_{i j}\right) \theta_{i} \theta_{j}\left(1-2 \lambda \theta_{t}\right)\right]^{1 / 2}} \\
& \quad(i \neq j \neq t)
\end{aligned}
$$

Details for the computation of the $\Gamma_{i j}$ 's have been given previously. ${ }^{9}$

With the above definitions, the equation of state in terms of reduced variables has the form $^{8}$

$$
\frac{\tilde{P} \tilde{v}}{\tilde{T}}=\frac{\tilde{v}^{1 / 3}}{\tilde{v}^{1 / 3}-1}-\frac{1}{\tilde{v} \widetilde{T}}
$$

where

$$
\tilde{P}=\frac{P}{P^{*}}
$$

and

$$
\tilde{T}=\frac{T}{T^{*}}
$$

The chemical potential is obtained from

$$
\mu_{1}=\left(\frac{\partial \ln Q_{N}}{\partial N_{1}}\right)_{T, V, N_{j \neq 1}}
$$

The activity $a_{1}$ of component 1 is then given by:

$$
\begin{aligned}
\ln a_{1}= & \frac{\mu_{1}-\mu_{1}{ }^{0}}{R T} \\
= & \ln \phi_{1}+\left(1-\frac{r_{1}}{r_{2}}\right) \phi_{2}+\left(1-\frac{r_{1}}{r_{3}}\right) \phi_{3} \\
& +\frac{P_{1}{ }^{*} r_{1} v^{*}}{R T}\left[3 \tilde{T}_{1} \ln \frac{\tilde{v}_{1}^{1 / 3}-1}{\tilde{v}^{1 / 3}-1}\right. \\
& \left.+\left(\frac{1}{\tilde{v}_{1}}-\frac{1}{\tilde{v}}\right)\right]+\frac{Z q_{1}}{2} \ln \Gamma_{11}
\end{aligned}
$$

Comparison of eq 27 with eq 8 of ref 1 gives for the $\chi_{1,23}$ parameters with $\phi_{23}=\phi_{2}+\phi_{3}$

$$
\begin{aligned}
\chi_{1,23}= & \frac{1}{\phi_{23}^{2}}\left\{\frac{Z q_{1}}{2} \ln \Gamma_{11}+\frac{P_{1}{ }^{*} r_{1} v^{*}}{R T}\right. \\
& \left.\times\left[3 \widetilde{T}_{1} \ln \frac{\tilde{v}_{1}^{1 / 3}-1}{\tilde{v}^{1 / 3}-1}+\left(\frac{1}{\tilde{v}_{1}}-\frac{1}{\tilde{v}}\right)\right]\right\}
\end{aligned}
$$

In the random case (zeroth approximation) the first term in eq 28 should be replaced by

$$
\begin{gathered}
\frac{v^{*}}{\tilde{v R T \phi_{23}^{2}}}\left(r_{1} X_{12} \theta_{2}\left(1-\theta_{1}\right)+r_{1} X_{13} \theta_{3}\left(1-\theta_{1}\right)\right. \\
\left.-\frac{Z q_{1}}{Z q_{2}} r_{2} X_{23} \theta_{2} \theta_{3}\right)
\end{gathered}
$$

Equation 28 is valid for binaries as well. ${ }^{9}$

The treatment of polymer-polymer-solvent systems with the above formulation involves the following three steps:

-Determination of the $X_{12}$ parameter from data on the Flory-Huggins $\chi_{12}$ interaction 
Table I. Equation of state parameters for pure components

\begin{tabular}{|c|c|c|c|c|}
\hline Pure component & $\frac{P^{*}}{\mathrm{~atm}}$ & $\frac{T^{*}}{\mathrm{~K}}$ & $\frac{v_{\mathrm{sp}}^{*}}{\mathrm{~cm}^{3} \mathrm{~g}^{-1}}$ & $\begin{array}{l}\text { Refer- } \\
\text { ence }\end{array}$ \\
\hline Benzene $\left(25^{\circ} \mathrm{C}\right)$ & 6194 & 4709 & 0.8860 & 13 \\
\hline Benzene $\left(58^{\circ} \mathrm{C}\right)$ & 6054 & 4829 & 0.8948 & 14 \\
\hline Polystyrene & 5395 & 7420 & 0.8100 & 15 \\
\hline Poly(vinyl methyl ether) & 5390 & 7201 & 0.8284 & 16 \\
\hline$n$-Pentane & 4010 & 4158 & 1.1828 & 17 \\
\hline Polyisobutylene & 4418 & 7580 & 0.9493 & 17 \\
\hline$n$-Heptadecane & 4418 & 5760 & 1.063 & 8 \\
\hline Chloroform & 6274 & 4450 & 0.5124 & 7 \\
\hline Poly(vinyl chloride) & 8645 & 7960 & 0.6240 & 18 \\
\hline Poly( $\varepsilon$-caprolactone $)$ & 5905 & 6363 & 0.769 & 18 \\
\hline Carbon tetrachloride & 5586 & 4700 & 0.487 & 18 \\
\hline Poly(ethylene oxide) & 6628 & 6469 & 0.7532 & 14 \\
\hline$n$-Tetracosane & 4542 & 6020 & 1.0544 & 8 \\
\hline
\end{tabular}

parameter for the system: solvent-polymer 2.

-Determination of the $X_{13}$ parameter from data on the Flory-Huggins $\chi_{13}$ interaction parameter for the system: solvent-polymer 3.

- Use of $X_{12}$ and $X_{13}$ for the determination of $X_{23}$ parameter from data on $\chi_{1,23}$ parameter for the system: solvent-polymer 2-polymer 3.

The pure component equation of state parameters are presented in Table I. Although the pure component parameters may change with temperature, the limited information available does not allow to consider this effect. In Figure 1 experimental and calculated values of the $\chi_{1,23}$ parameter are presented for the system chloroform (1)-polystyrene (2)poly(vinyl methyl ether) (3) at $25^{\circ} \mathrm{C}$. The density of chloroform at $25^{\circ} \mathrm{C}$ is ${ }^{19} 1.4794$ $\mathrm{g} \mathrm{cm}^{-3}$. The values of $X_{i j}$ parameters for this system are: $X_{12}=-2.27 \mathrm{calcm}^{-3}, X_{13}=$ $-16.19 \mathrm{cal} \mathrm{cm}^{-3}, X_{23}=0.015 \mathrm{cal} \mathrm{cm}^{-3}$. No substantial improvement has been found by introducing a second parameter with the treatment as suggested previously. ${ }^{9}$

In Figure 2, experimental and calculated

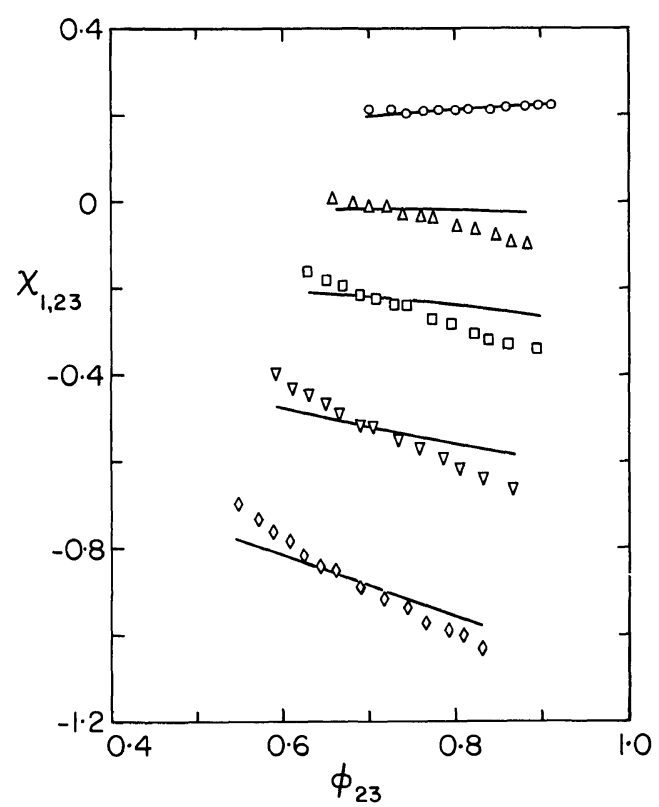

Figure 1. Experimental and calculated $\chi_{1,23}$ parameters versus polymer mixture segment fraction $\phi_{23}$ for the system chloroform (1)-polystyrene (2)-poly(vinyl methyl ether) (3) at $25^{\circ} \mathrm{C}$. points $\left(w_{2 p}\right): \bigcirc, 1.0 ; \triangle, 0.8012 ; \square, 0.6023 ; \nabla, 0.3337$; $\diamond, 0.0$.

values of the $\chi_{1,23}$ parameter are presented for the system benzene (1)-polystyrene (2)poly(vinyl methyl ether) (3) at $25^{\circ} \mathrm{C}$. The specific volume of benzene at $25^{\circ} \mathrm{C}$ is ${ }^{13} 1.1444$ $\mathrm{cm}^{3} \mathrm{~g}^{-1}$. The values of the $X_{i j}$ parameters for this system are: $X_{12}=0.13 \mathrm{calcm}^{-3}, X_{13}=$ $-1.20 \mathrm{calcm}^{-3}, X_{23}=0.019 \mathrm{calcm}^{-3}$. Once again, no substantial improvement has been found by using a second parameter. The interesting result is the surprising good agreement in the $X_{23}$ parameter obtained from data using two different probes. A minor difference in $X_{23}$ 's arising from the different size of the two probes, as indicated by the defining eq 19 , is always expected.

In Figure 3, experimental and calculated values of the $\chi_{1,23}$ parameter are presented for the system carbon tetrachloride (1)-poly(vinyl chloride) (2)-poly( $\varepsilon$-caprolactone) (3) at $65^{\circ} \mathrm{C}$. The specific volume of carbon tetrachloride is 
Thermodynamics of Polymer-Solvent Systems II.

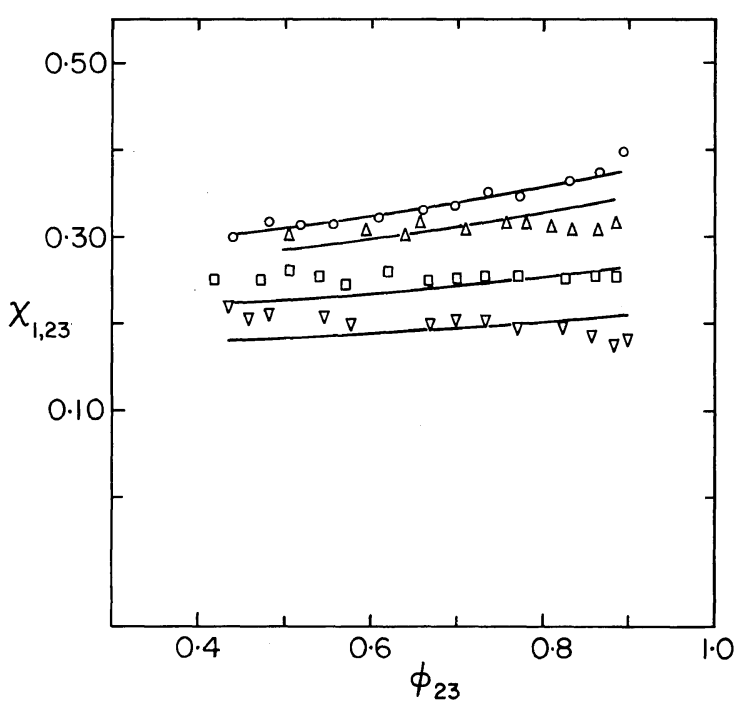

Figure 2. Experimental and calculated $\chi_{1,23}$ parameters versus polymer mixture segment fraction $\phi_{23}$ for the system benzene (1)-polystyrene (2)-poly(vinyl methyl ether) (3) at $25^{\circ} \mathrm{C}$. - , calculated; experimental points $\left(w_{2 p}\right): \bigcirc, 1.0 ; \triangle, 0.8012 ; \square, 0.3337 ; \nabla$, 0.0 .

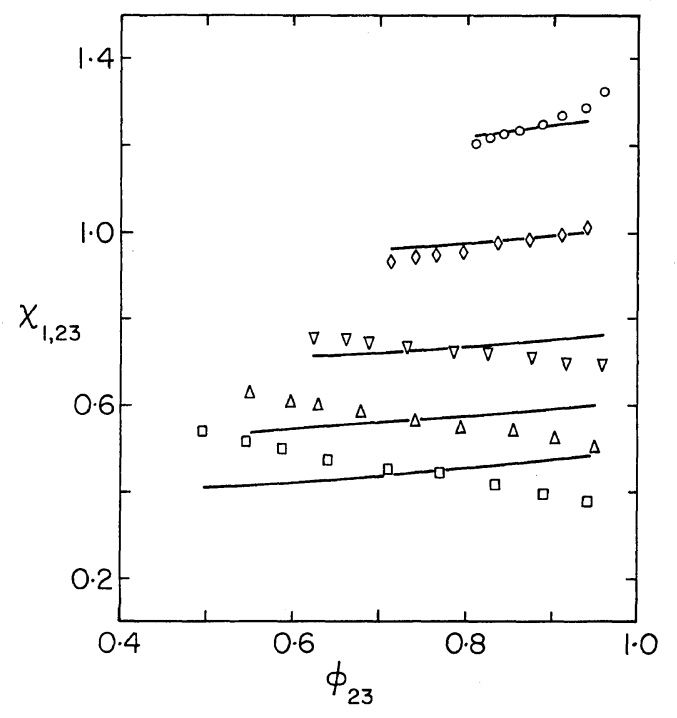

Figure 3. Experimental and calculated $\chi_{1,23}$ parameters versus polymer mixture segment fraction $\phi_{23}$ for the system carbon tetrachloride (1)-poly(vinyl chloride) (2)-poly( $\varepsilon$-caprolactone) (3) at $65^{\circ} \mathrm{C}$. - , calculated; experimental points $\left(w_{2 p}\right): \bigcirc, 1.0 ; \diamond, 0.7653 ; \nabla, 0.4784$; $\triangle, 0.2171 ; \square, 0.0$.

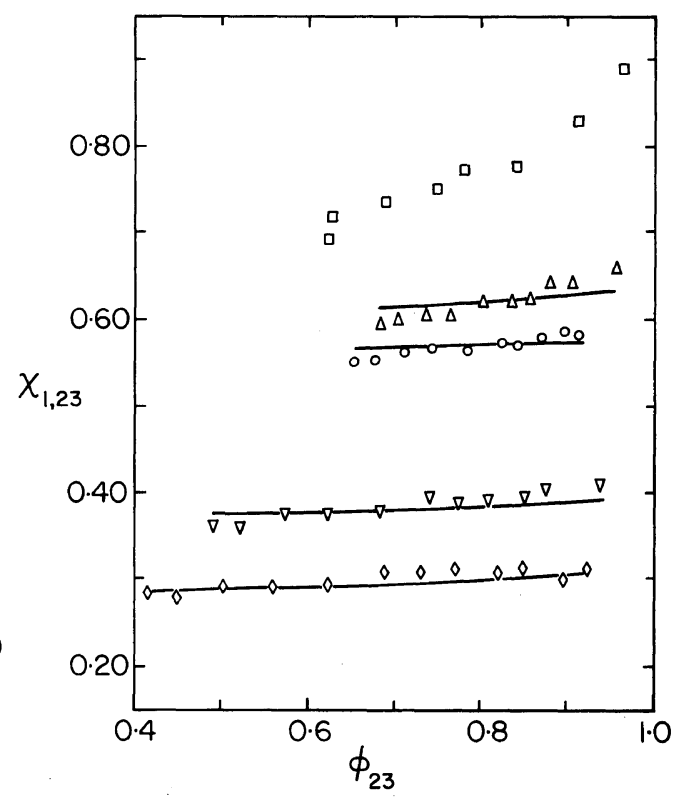

Figure 4. Experimental and calculated $\chi_{1,23}$ parameters versus polymer mixture segment fraction $\phi_{23}$ for the system $n$-pentane (1)-polyisobutylene (2)-nheptadecane (3) at $25^{\circ} \mathrm{C}$. — , calculated; experimental: $\square, n$-pentane-polyisobutylene molecular weight $40000^{17}$; $\triangle$, n-pentane-polyisobutylene molecular weight 2700 ; $O$, n-pentane-polyisobutylene molecular weight 1350 ; $\nabla, n$-pentane-polymer mixture, $w_{2 p}=0.3854 ; \diamond, n$ pentane- $n$-heptadecane.

taken as $^{20} 0.6639 \mathrm{~cm}^{3} \mathrm{~g}^{-1}$. The values of the $X_{i j}$ parameters for this system are $X_{12}=7.99$ cal cm ${ }^{-3}, \quad X_{13}=3.32 \quad \mathrm{cal} \mathrm{cm}^{-3}, \quad X_{23}=1.59$ cal $\mathrm{cm}^{-3}$. Olabisi, ${ }^{21}$ from gas-liquid chromatography measurements, has calculated $X_{23}$ at $120^{\circ} \mathrm{C}$ for this system to be $2.2 \mathrm{cal} \mathrm{cm}^{-3}(30 \%$ poly(vinyl chloride) per weight) and 3.0 cal cm $\mathrm{cm}^{-3}(50 \%$ poly(vinyl chloride) $)$. In view of the difference in temperatures, the reported values in the two works may be considered in satisfactory agreement with each other.

In Figure 4, the experimental and calculated values of the $\chi_{1,23}$ parameter are presented for the system: $n$-pentane (1)-polyisobutylene (2)$n$-heptadecane (3) at $25^{\circ} \mathrm{C}$. In the same figure data on $\chi_{12}$ parameter for the system pentanehigh molecular weight polyisobutylene ${ }^{17}$ are presented for the sake of comparison. The specific volume of pentane at $25^{\circ} \mathrm{C}$ is ${ }^{17} 1.6094$ 
$\mathrm{cm}^{3} \mathrm{~g}^{-1}$. The values of the $X_{i j}$ parameters for this system are: $X_{12}=-0.12 \mathrm{cal} \mathrm{cm}^{-3}, X_{13}=$ $0.83 \mathrm{cal} \mathrm{cm}^{-3}, X_{23}=-0.31 \mathrm{cal} \mathrm{cm}^{-3}$. The calculated line for the polyisobutylene sample of molecular weight 2700 was obtained with $X_{12}=0.49 \mathrm{cal} \mathrm{cm}^{-3}$. Discrepancies may be expected because of possible "end effects" in this range of molecular weights.

\section{BLOCK COPOLYMER-SOLVENT SYSTEM}

In this section the nonrandom new Flory theory is extended to the case of block copolymer-solvent systems. The treatment is limited to the case of two different types of blocks containing units of types A and B. Each block may have any number of units of a single type and the larger the blocks the better the approximation of the model is likely to be.

In the following treatment subscript 1 indicates the solvent containing units $\mathrm{S}$; subscript 2 indicates the block copolymer containing units $\mathrm{A}$ and $\mathrm{B}$; subscript 3 indicates the model homopolymer containing only units $A$; subscript 4 indicates the model homopolymer containing only units B. $r_{\mathrm{LM}}$ is the number of units of type $\mathrm{L}$ in the molecule of type $\mathrm{M}$. Thus, L may be $\mathrm{S}, \mathrm{A}$ or $\mathrm{B}$ and $\mathrm{M}$ may be 1,2 , 3 , or 4 .

Following Flory's convention ${ }^{8}$ the size of each unit (segment) will be set equal to the size of the solvent. For the solvent it will be set

$$
r_{1}=r_{\mathrm{S} 1}=1
$$

with a total number of external contacts per molecule:

$$
Z q_{1}=Z
$$

The total number of units in molecule 2 is

$$
r_{2}=r_{\mathrm{A} 2}+r_{\mathrm{B} 2}
$$

With a corresponding number of external contacts

$$
Z q_{2}=(Z-2) r_{2}+2
$$

The number of external contacts in which units A of the molecule 2 are participating is

$$
Z q_{\mathrm{A} 2}=(Z-2) r_{\mathrm{A} 2}+l
$$

where $l=0$ or 1 or 2 depending on how many times block of type $\mathrm{A}$ is an end block. The rest of the external contacts of molecule 2 are then

$$
Z q_{\mathrm{B} 2}=Z q_{2}-Z q_{\mathrm{A} 2}
$$

The fraction of contact sites $\mathrm{A}$ on each copolymer molecule is

$$
v_{\mathrm{A} 2}=\frac{Z q_{\mathrm{A} 2}}{Z q_{2}}
$$

and similarly for contact sites B

$$
v_{\mathrm{B} 2}=\frac{Z q_{\mathrm{B} 2}}{Z q_{2}}=1-v_{\mathrm{A} 2}
$$

In the solvent-block copolymer system there are two types of molecular surface fractions, namely

$$
\theta_{1}=\frac{N_{1} Z q_{1}}{N_{1} Z q_{1}+N_{2} Z q_{2}}=\frac{N_{1} Z q_{1}}{N Z q}
$$

and

$$
\theta_{2}=\frac{N_{2} Z q_{2}}{N Z q}=1-\theta_{1}
$$

The three types of unit surface fractions are

$$
\begin{gathered}
\Theta_{\mathrm{A}}=\frac{N_{2} Z q_{\mathrm{A} 2}}{N Z q}=\frac{N_{2} Z q_{2}}{N Z q} v_{\mathrm{A} 2}=\theta_{2} v_{\mathrm{A} 2} \\
\Theta_{\mathrm{b}}=\frac{N_{2} Z q_{\mathrm{B} 2}}{N Z q}=\frac{N_{2} Z q_{2}}{N Z q} v_{\mathrm{B} 2}=\theta_{2} v_{\mathrm{B} 2} \\
\Theta_{\mathrm{S}}=\frac{N_{1} Z q_{1}}{N Z q}=\theta_{1}=1-\Theta_{\mathrm{A}}-\Theta_{\mathrm{B}}
\end{gathered}
$$

If the total number of external contact pairs $\mathrm{L}-\mathrm{K}$ in the system is $N_{\mathrm{LK}}$, the local unit surface fractions are defined by the relations:

$$
\begin{aligned}
& N_{\mathrm{AA}}=\frac{N Z q}{2} \Theta_{\mathrm{A}} \Theta_{\mathrm{AA}} \\
& N_{\mathrm{BB}}=\frac{N Z q}{2} \Theta_{\mathrm{B}} \Theta_{\mathrm{BB}}
\end{aligned}
$$




$$
\begin{gathered}
N_{\mathrm{SS}}=\frac{N Z q}{2} \Theta_{\mathrm{S}} \Theta_{\mathrm{SS}} \\
N_{\mathrm{AB}}=N Z q \Theta_{\mathrm{A}} \Theta_{\mathrm{BA}}=N Z q \Theta_{\mathrm{B}} \Theta_{\mathrm{AB}} \\
N_{\mathrm{AS}}=N Z q \Theta_{\mathrm{A}} \Theta_{\mathrm{SA}}=N Z q \Theta_{\mathrm{S}} \Theta_{\mathrm{AS}} \\
N_{\mathrm{BS}}=N Z q \Theta_{\mathrm{B}} \Theta_{\mathrm{SB}}=N Z q \Theta_{\mathrm{S}} \Theta_{\mathrm{BS}}
\end{gathered}
$$

From the principle of conservation of the external contacts, ${ }^{7}$ the above definitions lead to the following relation:

$$
\sum_{\mathrm{L}} \Theta_{\mathrm{LK}}=1 \quad \mathrm{~L}, \mathrm{~K}=\mathrm{A}, \mathrm{B}, \mathrm{S}
$$

The nonrandom factors $\Gamma_{\mathrm{LK}}$ are defined in a manner analogous to that described in the first section. For example

$$
\Theta_{\mathrm{AB}}=\Theta_{\mathrm{A}} \Gamma_{\mathrm{AB}}
$$

With the above nomenclature, the exponential term of the partition function may be written as:

$$
-\frac{E_{0}}{v R T}=\frac{N Z q}{2 v R T}\left[\sum_{\mathrm{L}} \Theta_{\mathrm{L}} \varepsilon_{\mathrm{LL}}-\sum_{\mathbf{L}} \sum_{\mathrm{K} \neq \mathrm{L}}^{\prime} \Theta_{\mathrm{L}} \Theta_{\mathrm{KL}} \Delta \varepsilon_{\mathrm{LK}}\right]
$$

Where, in the sum $\Sigma^{\prime}$ each pair should be taken once, and

$$
\Delta \varepsilon_{\mathrm{LK}}=\varepsilon_{\mathrm{LL}}+\varepsilon_{\mathrm{KK}}-2 \varepsilon_{\mathrm{LK}}
$$

$$
\text { ( } \mathrm{L}, \mathrm{K} \text { being } \mathrm{A} \text { or } \mathrm{B} \text { or } \mathrm{S} \text { ) }
$$

The determination of the local unit fractions is done in exactly the same manner as it was done previously in the first section for the ternary case. Similarly the quasi-chemical conditions (eq 16), written in terms of local unit surface fractions, have the general form

$$
\frac{\Theta_{\mathrm{AA}} \Theta_{\mathrm{BB}}}{\Theta_{\mathrm{AB}} \Theta_{\mathrm{BA}}}=\exp \frac{\Delta \varepsilon_{\mathrm{AB}}}{v R T}=G_{\mathrm{AB}}
$$

Equation 22 is modified in a similar manner. The segment fractions are given by eq 9 for the case of two components $(i=1,2)$. The characteristic equation of state parameters are defined by the relations

$$
P^{*} v^{* 2}=\frac{Z q}{2 r}\left(\sum_{\mathrm{L}} \Theta_{\mathrm{L}} \varepsilon_{\mathrm{LL}}-\sum_{\mathrm{L}} \sum_{\mathrm{K} \neq \mathrm{L}}^{\prime} \Theta_{\mathrm{L}} \Theta_{\mathrm{KL}} \Delta \varepsilon_{\mathrm{LK}}\right)
$$

where

$$
N r=N_{1} r_{1}+N_{2} r_{2}
$$

Again,

$$
P^{*} v^{*}=c R T^{*}
$$

where now

$$
c=\phi_{1} c_{1}+\phi_{2} c_{2}=\phi_{1} c_{1}+\phi_{2}\left(\frac{r_{\mathrm{A} 2} c_{3}+r_{\mathrm{B} 2} c_{4}}{r_{2}}\right)
$$

The inherent assumption in eq 55 is that each unit has the same value for the Prigogine $c$ parameter, ${ }^{11}$ in both the homopolymer and in the block copolymer, which is likely to be true if the blocks are long enough.

With all these definitions the equation for the $\chi_{12}$ interaction parameter takes the simple form

$$
\begin{aligned}
\chi_{12}= & \frac{1}{\phi_{2}{ }^{2}}\left\{\frac{Z q_{1}}{2} \ln \Gamma_{\mathrm{SS}}+\frac{P_{1}{ }^{*} r_{1} v^{*}}{R T}\right. \\
& \left.\times\left[3 \tilde{T}_{1} \ln \frac{\tilde{v}_{1}^{1 / 3}-1}{\tilde{v}^{1 / 3}-1}+\left(\frac{1}{\tilde{v}_{1}}-\frac{1}{\tilde{v}}\right)\right]\right\}
\end{aligned}
$$

which is formally identical to eq 28 .

Application to the System: Benzene- $\alpha, \omega-$ Alcoxy Poly (ethylene oxide)

Data previously reported ${ }^{1}$ will be used to estimate the polyethylene (high $n$-alkane)poly(ethylene oxide) interaction constant. Each unit of the end blocks $\left(\mathrm{CH}_{3}\left(\mathrm{CH}_{2}\right)_{17}\right)$ and $\left(\mathrm{CH}_{3}\left(\mathrm{CH}_{2}\right)_{20}\right)$ is assumed to behave as a similar unit in $n$-tetracosane, while each unit of the internal block is assumed to behave as a similar unit in the poly(ethylene oxide) E600M ${ }^{1}$ sample. The reduced volume for benzene at $55^{\circ} \mathrm{C}$ is ${ }^{14,20} 1.3282$.

From the treatment of the data on benzene (S) E600M (A) system, the following values are obtained: 


$$
\begin{aligned}
& \varepsilon_{\mathrm{AS}}=166350 \mathrm{cal} \mathrm{cm}^{-3} \quad \text { and } \\
& X_{\mathrm{SA}}=-0.75 \mathrm{cal} \mathrm{cm}^{-3}
\end{aligned}
$$

Similarly, for the system benzene (S)- $n$-tetracosane (B),

$$
\begin{aligned}
& \varepsilon_{\mathrm{BS}}=132706 \mathrm{cal} \mathrm{cm}^{-3} \quad \text { and } \\
& X_{\mathrm{SB}}=6.03 \mathrm{cal} \mathrm{cm}^{-3}
\end{aligned}
$$

Using these binary parameters, the treatment of the data on benzene-block copolymer 1845-18 ${ }^{1}$ gives

$$
\begin{aligned}
& \varepsilon_{\mathrm{AB}}=160390 \mathrm{cal} \mathrm{cm}^{-3} \quad \text { and } \\
& X_{\mathrm{BA}}=-3.84 \mathrm{cal} \mathrm{cm}^{-3}
\end{aligned}
$$

and, similarly, for the system benzene-block copolymer $21-45-21^{1}$

$$
\begin{aligned}
& \varepsilon_{\mathrm{AB}}=160368 \mathrm{cal} \mathrm{cm}^{-3} \quad \text { and } \\
& X_{\mathrm{BA}}=-3.23 \mathrm{cal} \mathrm{cm}^{-3}
\end{aligned}
$$

The surprisingly negative values for the $X_{\mathrm{BA}}$ parameter may be due to end effects. In order to test this hypothesis, each unit of the internal block has been simulated with a similar unit of the high molecular weight poly(ethylene oxide) molecule (see ref 1).

For this system, the following values have been obtained at $70^{\circ} \mathrm{C}$ :

$$
\begin{aligned}
& \varepsilon_{\mathrm{AS}}=169134 \mathrm{cal} \mathrm{cm}^{-3} \quad \text { and } \\
& X_{\mathrm{SA}}=0.97 \mathrm{cal} \mathrm{cm}^{-3}
\end{aligned}
$$

Using this parameter, the system benzene-(1845-18) gave

$$
\begin{aligned}
& \varepsilon_{\mathrm{AB}}=159395 \mathrm{cal} \mathrm{cm}^{-3} \quad \text { and } \\
& X_{\mathrm{BA}}=4.54 \mathrm{cal} \mathrm{cm}^{-3}
\end{aligned}
$$

which seem to be more reasonable values for this type of pair interaction.

In Figure 5 experimental and calculated data are presented for the solvent-copolymer systems and the corresponding solventhomopolymer systems. In view of the several oversimplistic assumptions in this rather elementary theory, the results may be considered

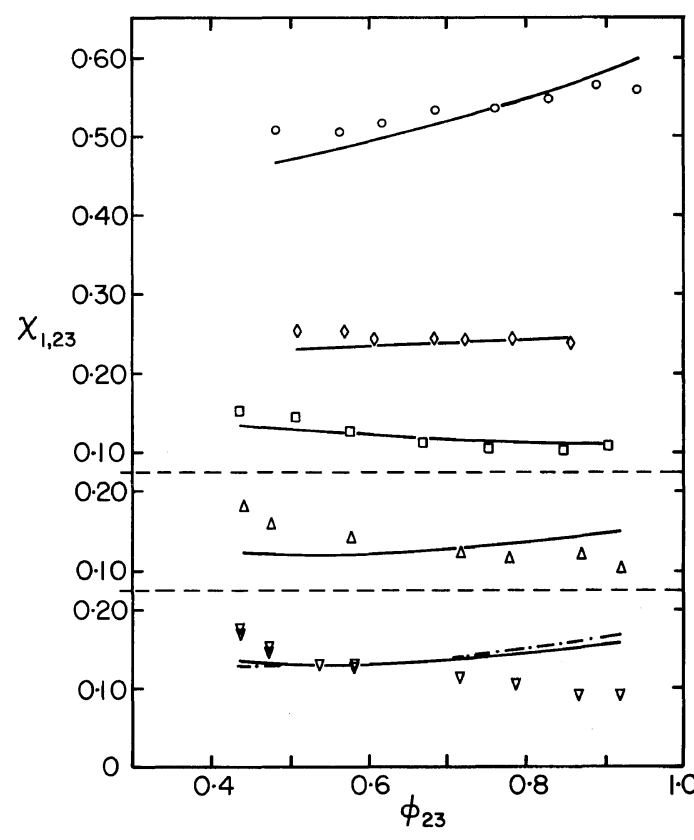

Figure 5. Experimental and calculated $\chi_{1,23}$ parameters versus polymer mixture segment fraction $\phi_{23}$ for the system benzene (1)-poly(ethylene oxide) (2)- $n$ tetracosane (3). $55^{\circ} \mathrm{C}$; ----, calculated with benzene-poly(ethylene oxide) parameters at $70^{\circ} \mathrm{C}$; experimental: $\bigcirc$, benzene- $n$ tetracosane at $55^{\circ} \mathrm{C} ; \diamond$, benzene-poly(ethylene oxide) molecular weight 100000 at $70^{\circ} \mathrm{C} ; \square$, benzenepoly(ethylene oxide) molecular weight 600 at $55^{\circ} \mathrm{C} ; \triangle$, benzene-copolymer $21-45-21$ at $55^{\circ} \mathrm{C} ; \nabla$, benzenecopolymer $18-45-18$ at $55^{\circ} \mathrm{C}$.

as gratifying.

\section{CONCLUSIONS}

In this paper we have presented one of the many ways in which data on the FloryHuggins $\chi$ interaction parameters for ternary systems may be treated with the new Flory theory. To our knowledge this is the first attempt to treat this type of data (nonzero solvent concentration) with an equation of state approach. The introduction of the $\chi_{1,23}$ parameter leads to a uniform treatment of ternary data for polymer solutions. Extension of the treatment to more than three components is straightforward. In this work only 
one binary parameter $\left(\varepsilon_{i j}\right)$ has been used for fitting the data, although previous experience ${ }^{7}$ has shown that just one parameter may not be enough for treating data on other thermodynamic functions of mixing such as heats of mixing and/or volumes of mixing. Due to the lack of more complete information, the binary parameters were determined using only data on $\chi$ interaction parameters. Since this information may not be enough to give unique values of the binary parameters, the values of the parameters obtained in this work should be considered with caution. As for the fit of the data, before a final judgement is passed on the validity of the theory, the approximate nature of the pure component equation of state parameters of Table I should be considered, since variations of these parameters with temperature and molecular weight for oligomers and polymers have not been taken into account in this work. Probably one of the merits of this simple approach is its generality. More data and better pure component parameters are necessary to give a more definite judgement on the validity of the approach.

Acknowledgements. The authors are grateful to NSERC for financial support.

\section{NOTATION}

\footnotetext{
$a_{1}=$ activity of component 1 (solvent)

$E_{0} \quad=$ mean intermolecular potential energy

$G_{i j} \quad=$ binary constant defined by eq 16

$g_{\mathrm{c}}=$ random combinatorial term

$g_{\mathrm{NR}}=$ nonrandom combinatorial factor

$l=$ number indicating how many times a particular block is an end block

$N=$ number of moles

$N_{i j} \quad=$ total number of external contacts of type $i-j$

$P \quad=$ pressure

$Q_{\mathrm{N}} \quad=$ configurational partition function

$R \quad=$ gas constant

$r \quad=$ number of segments per molecule

$3 r c=$ equivalent number of translational degrees of freedom per molecule

$T=$ temperature $(\mathrm{K})$

$v \quad=$ volume per segment

$w_{2 p}=$ weight fraction of component 2 in the polymer mixture
}

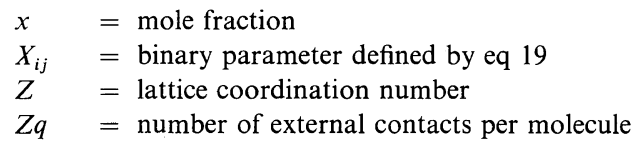

Greek Letters

$\Gamma \quad=$ nonrandom quasichemical factor

$\gamma=$ geometric factor in the cell partition function

$\Delta \quad=$ property difference

$\varepsilon_{i j} \quad=$ interaction energy parameter for contact of type $i-j$

$\Theta \quad=$ unit surface fraction

$\Theta_{\mathrm{LK}}=$ local unit surface fraction for units of type $\mathrm{L}$ around units of type $\mathrm{K}$

$\theta \quad=$ molecular surface fraction

$\theta_{i j} \quad=$ local molecular surface fraction for molecule of type $i$ around molecule of type $j$

$\lambda=$ nonrandom auxiliary parameter

$\mu \quad=$ chemical potential

$\mu_{1}^{0}=$ chemical potential of pure component 1

$v_{\mathrm{LM}}=$ fraction of unit contacts of type $\mathrm{L}$ in a molecule of type $\mathbf{M}$

$\phi_{i} \quad=$ segment fraction of component $i$

$\chi_{i j}=$ Flory-Huggins interaction parameter

$\chi_{1,23}=$ quantity defined in ref 1

Superscripts

* $\quad=$ reducing quantities

$\sim \quad=$ reduced quantities

$0 \quad=$ random values

Subscripts

$\begin{array}{ll}i & =\text { quantity pertaining to component } i \\ \mathrm{~L} & =\text { quantity pertaining to unit } \mathrm{L} \\ \mathrm{LM} & =\text { quantity pertaining to unit } \mathrm{L} \text { in component } \mathrm{M}\end{array}$

\section{REFERENCES}

1. C. Panayiotou and J. H. Vera, Polym. J., 16, 89 (1984).

2. D. R. Paul and S. Newman, Eds., "Polymer Blends," Academic Press, New York, 1978.

3. O. Olabisi, L. M. Robeson, and M. T. Shaw, "Polymer-Polymer Miscibility," Academic Press, New York, 1979.

4. P. de Saint-Romain, Ph. D. thesis, Chemistry, McGill University, Montreal, 1977.

5. J. A. R. Renuncio and J. M. Prausnitz, Macromolecules, 9, 898 (1976).

6. R. H. Lacombe and I. C. Sanchez, J. Phys. Chem., 80, 2568 (1976).

7. C. Panayiotou and J. H. Vera, Fluid Phase Equil., 5, 55 (1980).

8. P. J. Flory, R. A. Orwoll, and A. Vrij, J. Am. Chem. Soc., 86, 3507 (1964).

9. C. Panayiotou and J. H. Vera, Canadian J. Chem. 


\section{Panayiotou and J. H. Vera}

Eng., 59, 501 (1981).

10. P. J. Flory, J. Chem. Phys., 10, 51 (1942).

11. I. Prigogine (with the collaboration of A. Bellemans and V. Mathot), "The Molecular Theory of Solutions," Interscience, New York, 1957.

12. E. A. Guggenheim, "Mixtures," Clarendon Press, Oxford, 1952.

13. B. E. Eichinger and P. J. Flory, Trans. Faraday Soc., 64, 2035 (1968).

14. C. Booth and C. J. Devoy, Polymer, 12, 309 (1971).

15. H. Shih and P. J. Flory, Macromolecules, 5, 758
(1972).

16. A. Robard, Ph. D. thesis, Chemistry, McGill University, Montreal, 1978.

17. B. E. Eichinger and P. J. Flory, Trans. Faraday Soc., 64, 2066 (1968).

18. O. Olabisi, Macromolecules, 8, 316 (1975).

19. Landolt-Bornstein, "Zahlenwerte and Funktionen," II Band, 1 Teil, Springer-Verlag, Berlin, 1971.

20. J. Timmernans, "Physico-Chemical Constants of Pure Organic Compounds," Vol. I, American Elsevier, New York, 1950. 